\title{
En torno al concepto y ejercicio de la policía
}

\section{INTRODƯCCIÓN}

La Escuela de Policía municipal de Barcelona, creada por acuerdo del Ayuntamiento adoptado el 30 de julio de 1954, tiene por objeto seleccionar y formar a quienes aspiren a ingresar como Guardias del Cuerpo de Policia del Municipio, capacitar a los pertenecientes al mismo que pretendan ascender a las categorías superiores, hasta la de Sub-oficial inclusive, y perfeccionar a los Oficiales.

La misión de la Escuela puede extenderse a sus alumnos después de terminar la docencia, en forma de cordial comunicación, consejo e informaciones, conferencias y cursillos, préstamos gratuitos de libros y Revistas profesionales.

A los efectos docentes se divide la Escula en dos secciones: la de formación y la de capacitación y perfeccionamiento, según el Reglamento de $\mathbf{1 3}$ de mayo de $\mathbf{1 9 5 5 .}$

Tras las pruebas de acceso, se ingresa en la Escuela como alumno aspirante de la sección de formación, en la que se prepara a İos neófitos, se suscita en ellos el espíritu de sacrificio y se moldean profesionalmente para ser Guardias de la Policía smunicipal.

Tres períodos comprenden los estudios del curso teórico y

(") Coriferencia pronunciada en la Escuela de le Policía rasnicipa! de Barceioaa, eç motivo de $\frac{1}{2}$ enirega de placas y rombraziersos a los ziumnos de la zrissc5és promoción. 
práctico de formación, durante los cuales se deparan a los aspirantes lecciones de Historia, Gramática, Aritmética y Geometría; Toponimia de la Ciudad; Principios de la organización político-administrativa española, y Moral cívica; Organización local, Deberes comunes de los funcionarios y especiales de los pertenecientes al Cuerpo de Policía municipal, y Ordenanzas municipales de policía y de exacciones; Circulación en genera1, y tránsito urbano; Educación física, prestación de servicios propios del cargo y actuación profesional.

Quienes alcancen el diploma de aptitud podrán tomar parte en las pruebas de ascenso a plazas de Cabo, Sargento y Subof icial.

Para obtener ese preciado diploma se requiere aprobar los cursos de capacitación y perfeccionamiento correspondientes a cada categoría y nutridos por "enseñanzas teóricas” de formación moral, ética profesional, virtudes y caracteres del mando y Régimen de las Entidades locales; y por "enseñanzas prácticas" de adiestramiento para el ejercicio del mando y aplicaciones policiales de la educación física.

Bastarian estas sucintas referencias del Reglamento por el que se rige la Escuela, para comprender que su inspiración y los designios de su funcionamiento responden al concepto clásico de la Policía, considerada tradicionalmente como organización y reglamentación interna de un Estado para mantener el orden conforme a las leyes establecidas y mediante un Cuerpo encargado de vigilar el cumplimiento de las mismas, con el fin de alcanzar la debida cortesía y urbanidad en el trato y costumbres de la comunidad civil.

En este sentido inicial bien podemos ver cómo Cervantes consideró a Barcelona ciudad eminentemente policial, al denominarla "archivo de la cortesía", en cuanto ésta significa trato de atención, respeto y afecto que dispensa gracia o merced.

Paralela a la cortesía es la urbanidad, reflejo de buenos modos en cualquier ámbito de convivencia, por lo que urbanizar equivale primeramente a hacer urbano y sociable a alguien, y después, a convertir en poblado una porción de terreno o prepararfo para la edificación. 
A fines de 1872 publicaba D. Mociesto Fossas Pi, Arquitecto de la Real Academia de San Fernando y Académico correspondiente de la misma, su Tratado de policia y obras públicas urbanas, en el que hallamos conjugadas, siquiera sea de modo enunciativo, muestras precedentes interpretaciores, y donde no sería muy aventurado vislumbrar algunos remotos atisbos del moderno Régimen del Suelo y de Ordenación urbana; pues como se lee en la Orden de 13 de marzo de 1873, dictada por el Ministro de Fomento para declarar ell tratado de utilidad pública, éste vino a aclarar los deberes y derechos recíprocos de la Administración y de los propietarios y a establecer la perfecta inteligencia que entre ellos ha de existir, y además se ocupaba ya previsoramente de los proyectos, contratas y obras, edificios y terrenos de diversa índole, mercados, establecimientos de espectáculos públicos; obras de utilidad y uso públicos; expropiación forzosa, reforma, mejora y ensanche de poblaciones ; servidumbres de interés público, en las que acogía la alineación, aceras, alcantarillas y canalizaciones, embellecimiento de la' vía pública y permiso de construcción; limitaciones para edificar y respecto de los establecimientos incómodos, insalubres y peligrosos, etcétera.

Bien decía Macarel que la autoridad administrativa tiene unæ función general: la de proveer por medio de ordenanzas a la ejecución de las leyes, a la conservación del orden público y $\approx$. las diferentes necesidades de la sociedad, y dos funciones especiales: la de ejercer singular patrocinio en ciertos establecimientos públicos y la de administrar con esmero el patrimonio púbico.

Mas la palabra "autoridad" suele aplicarse unas veces al derecho de ordenación al bien común, y otras a la persona o personas que ejercen ese derecho. En el primer supuesto, la autoridad constituye uno de los elementos de la sociedad política, porque ia existencia de ésta, como la de las demás sociedades, no se concibe sin orden, ni éste se comprende sin poder de ordenación; y en el segundo supuesto, la autoridad es el sujeto que actúa el poder ordenador. Por eso distinguía $D$. Gonzalo del Castillo Alonso, siguiendo la corriente de ics escolásćicos, la "autoriáad moral" o sustancial, que representa el poder poilti- 
co, y la "autoridad operativa" o formal, que no es realmente sino mero atributo para la eficacia de aquélla o de su ejercicio dentro de la correspondiente órbita de competencia.

Las apuntadas derivaciones conceptuales comportan el supuesto de misión tutelar que hallaremos siempre y desde cualquier ángulo en que enfoquemos la (rfunción" de policía y, a la vez, implican la convergencia en el "órgano" de los cometidos asignados a los individuos de la milicia o Guardia urbana, institución al servicio de la Ciudad y de la urbe.

El "ơrden público» era para Hauriou, en cuanto afecta al sentido de la policía, orden material y exterior, considerando como un estado de hecho opuesto al desorden. Es decir, la paz contrapuesta a la perturbación. Discurriendo por esta vertiente, percibimos el orden como afirmación del buen dispositivo de la cosa pública frente a las perturibaciones alteradoras de la normalidad, y el idesorden viene a ser el síntoma que guía a la policía como la fiebre al médico. De ahí que aquélla y éste pretendan hacer desaparecer la anormalidad, aplicando inmediatamente una terapéutica de rigurosa eficacia, sin perjuicio de estudiar después las causas y remedios del malestar.

Ciertamente, determinar el concepto de policía en relación con el orden público equivale, en expresión de Garrido Falla, a considerar la primera, de una vez para siempre, como un concepto relativo cuya determinación sólo será posible tras un examen de las circunstancias que la condicionan; pues si lo que se entiende por orden público depende en cada momento de las ideas que prevalecen sobre el régimen político y los fines del Estado, de esto se deduce que las diferentes concepciones dominantes durante el siglo xix y en la etapa actual nos dan la medida de las variaciones experimentadas por la policía administrativa:

Precisemos, no obstante, una previa discriminación en torno a la acción administrativa, cuya finalidad genérica se orienta hacia la satisfacción de necesidades públicas, y en la esfera de la competencia municipal all gobierno y administración de los intereses peculiares de los pueblos. 


\section{La NOCIÓN de Policía}

Sin más que remitirnos a los artículos 101 y 102 de la Ley de Régimen local, encontraremos concreciones de fines que incumbe conseguir a los Municipios y, en su representación genuina, a los Ayuntamientos: unos son de Policia urbana y rural, extinción de incendios, salvamento, defensa pasiva, protección de personas y bienes, policía de construcción, fábricas, establecimientos mercantiles y espectáculos; otros, de fomento de la vivienda y del turismo, protección y defensa del paisaje, museos, monumentos artísticos e históricos, playas y balnearios; otros se refieren a los servicios de guardería rural, surtido de agua potable, alumbrado público, pavimentación, cementerios, limpieza viaria, destrucción o tratamiento técnico-sanitario de basuras y residuos, desinfección y desinsectación, botiquín de urgencia, asistencia médico-farmacéutica a familias desvalidas, inspección sanitaria de alimentos y bebidas.

Es decir, que para satisfacer las necesidades y proveer las atenciones que constituyen la primordial razón de ser del Municipio, se agrupan unas y otras bajo distintos dictados correlativos a los "medios" de que dispone la Administración local para llevar a cabo las misiones que le competen, tan ampliadas y elevadas por el vigente régimen, que contemplan el horizonte ilimitado de las aspiraciones ideales de la comunidad vecinal.

Aparte de la "legislación", con sus caracteres de generalidad e impersonalidad, como premisas de la igualdad jurídica, se nos ofrecen en la aludida trayectoria discriminadora tres modos de "administración" o pertenecientes a la actividad administrativa, que son los anteriormente subrayados: policia, fomenio y servicio público.

En términos de Jordana de Pozas, el "servicio público" es una modalidad de la acción administrativa, encaminada a satis. facer la necesidad públioa de que se trate de manera directa, por órganos de la propia Administración creados a! efecto y con exclusión o en concurrencia de los particulares.

La uacción de fomenton es sna vía media ọıe sigue la Admi- 
nistración para proteger o promover aquellas actividades, establecimientos o riquezas debidos a los particulares y que satisfacen necesidades públicas o se estiman de utilidad general, sin crear servicios públicos ni usar de la coacción.

La "actividad de policía” aparece cualificada, en la definición de Merkl, porque mediante la amenaza o el empleo de la coacción persigue la previsión o desviación de los peligros o perturbaciones del orden.

La noción de policía tiene, pues, capital importancia en campo del Derecho administrativo, dentro del cual acompasa diversos módulos y afecta a varios sectores de la vida municipal, tantos como cabe imaginar para conseguir el buen orden de las ciudades y repúblicas por la aplicación de las Ordenanzas establecidas para su mejor gobierno.

Con razón dice Fleiner que el concepto de "policía" ha seguilo una evolución accidentada. En Grecia, la voz politeia hubo de significar el ordenamiento o constitución de la Ciudad. En Roma, admite Hague que la politia comprendiera incluso un Cuerpo similar al de la Policía de hoy.

Hacia fines del siglo jurídico de Francia las palabras police y policité, alusivas a la ordenación, prosperidad y seguridad públicas que ha de procurar la Autoridad, bien entendido que esta idea deil orden no se apoya en la presión que desde fuera pueda ejercerse sobre la colectividad, sino en un equilibrio que se suscita en su interior.

El Traité de la Police de Mr. Nicolás de la Mare comenzó a publicarse en' París el año 1713 y se encaminaba, según su autor, "a la plus parfaite felicité dont il puisse jouir en cette vie". Esa felicidad humana dependía de tres clases de bienes: los del alma, los del: cuerpo y los que se llaman de fortuna; y para alcanzarla aconsejábase velar por lia religión y la disciplina de las costumbres, la salud pública, abastos, vías y viviendas, orden público, ciencia y arte, comercio, manufàcturas y artes mecánicas.

Superada la primera mitad del siglo xv comienza a barajarse en Alemania la palabra Polizei y el año 1758 publica en Gotinga Johannes Heinrich Gottlob von Justi su obra Grundsatze der Polizeizerssenschaft o Fundamentos de la Ciencia de la Policía, 
que comprende ias jeyes y regiamentos concernientes al interior de un Estado, dirigidas a a de sus fuerzas, contribuir a la felicidad de los súbditos..., hacerles la vida más cómoda, procurarles la's cosas que necesiten para sibsistir y, principalmente, conservar el orden y la disciplina.

Corresponde a mi querido maestro, D. Luis Jordana de Pozas, la aportación de ésta y otras interesantes noticias prácticamente desconocidas hasta que él las introdujo entre los estudiosos, en su atrayente conferencia "Los cultivadores españoles de ia Ciencia de la Policía", pronunciada en el Instituto de Estudios de Administración Local, el año 1941.

Para nosotros tiene singular interés destacar la obra y la figura del traductor de von Justi, D. Antonio Francisco Puig y Gelabert, Doctor en Sagrados cánones, perteneciente al Gremio y Claustro de la Universidad de Huesca, Abogado de la Real Audiencia de Cataluña y Juez de reclamaciones de la Curia Real ordinaria del Corregimiento de Barcelona, Director de Derecho público criminal de la Academia de Jurisprudencia teórico-práctica de nuestra Ciudad.

Con el propósito de facilitar a los pasantes que hubieran de someterse ail examen de Ciencia dei Gobierno, en sus tres ramos de Policía, Política y Economía, ordenado por la Real Audiencia y Principado, preparábase Puig y Gelaber para escribir un compendio de las principales obras españolas sobre Policía; pero desistió de ese proyecto al conocer el tratado del autor alemán, por la traducción francesa, y no sólo lo vertió al español, sino que lo avaló con notables aclaraciones y comentarios en los que se muestra creyente y respetuoso con las tradiciones y doctrina católicas, impugna la tesis de la libertad de conciencia, estima dañoso el divorcio y preferibles las Casas de acogimientó de madres desamparadas a los Hospitales de expósitos, e intangible el celibato eclesiástico.

También vislumbra Puig y Gelabert un Montepío general para socorrer a los trabajadores en casos de enfermedad, imposibilidad y muerte, con lo que, según observa el propio Jordana, anticipóse en ciento veinticinco años a otro ilustre cata!án, don José Maluques, f́ndacior dei Instituto Nacional de Previsión, 
y en siglo y medio al seguro tota' de nuestro Fuero del Trabajo, y reveló clara perceptibilidad del concepto de "seguridad social" propugnado modernamente por Beveridge.

La obra que glosamos, señala como fin de la policía el de afianzar al Estado por la sabiduría de sus reglamentos y aumentar su fuerza y poder tanto como sea posible.

El primer libro, dedicado a la cultura de las tierras, comprende lo referente a los cultivos, a la fundación y régimen de las ciudades y a la estética rural y urbana, así como lo que respecta a la población, en su triple aspecto inmigratorio, de aumento de los nacionales y de disminución de la mortalidad. EI segundo libro trata de la economía rural, de las manufacturas, oficios y profesiones, del comercio, el dinero y el crédito, y de los medios para que florezca la economía y quitar los obstáculos que lo impidan. El tercer libro se aplica a las costumbres de los súbditos y versa sobne la religión, el orden y disciplina de costumbres, la educación, el lujo y la ociosidad, y la seguridad interior del Estado, que abarca la justicia y la policía propiamente dicha, preventiva de los delitos y desórdenes.

Tomando la' palabra "policía" en su significado estricto, la función o actividad de tal índole se endereza a castigar los crímenes que ponen a los hombres fuera del cumplimiento de los deberes que la sociedad exige de ellos, y sin los cuales turban la quietud y la tranquilidad pública:

Afirmada esa radical tendencia sancionadora, surge la meditación sobre la fragilidad humana, y se nos dice que la policía no debe prohibir a los ciudadanos los divertimientos inocentes, como la danza, las comedias y otros semejantes..., pero sí ha de impedir el libertinaje y la impudicidad...; todo ello con tal mesura, que no extienda las cosas hasta su último rigor por no tener que abrir la puerta a males mayores.

Igualmente deben evitarse el escándalo, la crápula y la embriaguez..., los juramentos y las blasfemias..., los gritos, golpes y alborotos por las calles, sea de noche o de día, y en caso de que esto suceda, la Policía debe hacer prender a los delincuentes y entregarilos para que los lleven a la cárcel.

En cuanto a las disposiciones policia'es, se nos depara el ati- 
nado consejo de que sean corias, inteligibies y a vista de todo el mundo, para que cada uro sepa lo que aebe hacer y lo que ha de evicar, y así produzcan el efecto deseado.

Conviene que la Policía tenga siempre el ojo abierto sobre las facciones y las conspiraciones que se formen en el Reino y dé luego aviso de ellas..., y ha de obrar con discernimiento y sin faltar a la prudencia ni interpretar jamás desproporcionadamente los objetos ni los procederes de los súbditos..., pues siendo el brazo de que se sirve el Soberano para hacer ejercitar sus leyes y ordenanzas con el fin de conservar la seguridad pública, debe extremarse el cuidado en evitar todo lo que pueda perturbarla $y$ herirla.

Como puede verse, para los helenos la politeia equivalia all público gobierno o, en expresión de Aristóteles, al concierto y orden entre aquellos que moran en la Ciudad; para los romanos, como Cornelio Nepote, la politia consistía en seguir los usos de la Ciudad (fungi civitatis more); en Francia cifrábase la police, según de la Mare, en garder le bien public para procurar la felicidad humana; en Alemania la polizei se orientaba, conforme indica Mayer, al buen estado de la cosa común (guter Stand der Gemeinwessens), kasta que Pütter publicó en 1770 sus Institutiones izsis publici, donde advierte Garrido Falla, al comentarlas er 1954, la exclusión de la llamada "policía del bienestar" (Wohlfhrspolizei) del concepto astricto de policía, que se contrae así a una "policia de seguridad" (Sicherheitspolizei).

Entre nosotros, y por la versión que nos llegara de Puig $y$ Gelabert, la policía tiende a arreglar todas las cosas relativas ai estado presente de la Sociedad, afirmarla y mejorarla... por caminos llanos y seguros para el pueblo, de suerte que todo él sea industrioso y tenga destino de qué vivir proporcionado a sus fuerzas y talento, con orden y disciplina que garanticen la convivencia en la Ciudad.

De la morigeración en las costumbres, de ilas reglas correcsas de cortesía, discreción y urbanidad, se sigue, por tanto, el buer orden y guarda de las circiades, que se condensa en ei debido cumplimiento de lás leyes y ordenanzas.

Fste sentir y practicar, al que llamara $\mathrm{San}$ Pio V De polstica 
REVL-1957, núm. 93. BERMEJO GIRONES, JUAN IGNACIO. EN TORNO AL CONCEPTO Y EJERCI:.. vel civilis, y al que hoy denominamos "civismo", fué timbre glorioso de nuestra misión civilizadora en América, cuyos indigenas "diferían poco de fieras" - según relatan documentos de la época- hasta que la religión y el trato de los españoles ies enseñó la "policía".

Satisfactorio habrá de resultar, al presente, que lo que hoy llaman los norteamericanos police power o poder de policía, sea en puridad el fruto de la semilla que sembraron los hombres de Hispania, inspirados en el signo de la Cruz, portadores de la más avanzada cultura, esforzados en la milicia, transplantadores de sabias leyes e instituciones de gobierno local, y en quienes se hubo de admirar más tarde la "grandeza y majestad, trato y policía de sus gentes".

\section{Obediencia y disciplina}

Desde cualquier punto de mira que se enfoque la doctrina de los fines del Estado, observaremos que la organización del Poder público es fundamento ineluctable y universal de la vida, supuesto previo y cardinal de la comunidad política e idea subyacente, por tanto, en todas las teorías ensayadas e imaginables, y que la garantía de la libertad individual acucia los desvelos de los distintos pensadores y gobernantes.

Frente a los "arquitectos de negaciones", dice González García, hay que confiar en las pristinas fuentes de la vida para que discurran, de nuevo, por el mismo cauce la razón y los valores humanos; porque cuando se marchitan las grandes concepciones determinadas por la floración de las "verdades eternas", ta sociedad queda arrasada en sus bases e impera el frenesí anárquico de los individuos.

El Estado refuerza su voluntad de imperium para salvaguardar la suprema unidad de su conjunto ; es autor de sus actos, dispone de potestad para realizarlos con eficacia; tiene autoridad, poder o facultad, afirmaba Posada, para definir las leyes de su acción y cumplir su fin.

Se vuelve a entregar al gobernante un amplio poder y se 
acaricia un Derecho administrativo ampliamente amparado en la "discreción" respecto a los "asuntos de policía", subraya $\mathrm{Pi}$ Suñer, un poco a la manera del clásıco von Justi y sus discípulos, cuyas viejas páginas revelan un espíritu de creación que la corriente de los años no ha conseguido sepultar.

Así acontece en la esfera de la Administración local, donde el Alcalde tiene la máxima prestancia representativa y se le atribuye competencia para ejercer todas las facultades de gobierno y administración no reservadas "expresamente" al Ayuntamiento pleno o a la Comisión permanente, además de las que ostenta de modo singular y peculiar, entre las cuales importa destacar estas:

a) aplicar las Ordenanzas de gobierno, construcción, policía urbana, servicios especiales y exacciones, y los Reglamentos municipales ;

b) hacer que se cumplan las leyes y disposiciones gubernativas;

c) conceder licencias de apertura de establecimientos fabriles, industriales o comenciales y de cualquier otra índole;

d) dirigir e inspeccionar la policía urbana, rural, sanitaria, de subsistencias, de seguridad y circulación, y de costumbres, publicando al efecto bandos, órdenes, circulares e instrucciones;

e) reprimir y castigar las faltas por desobediencia a su Autoridad y las infracciones de las Ordenanzas y Reglamento municipales;

f) mantener el orden y proveer a la seguridad pública e individual ;

g) adoptar personalmente y bajo su responsabilidad, 'en caso de gravedad producida por epidemia, trastornos de orden público, guerra, inundación o cualquier otro accidente análogo, las medidas que juzgue necesarias, dando cuenta inmediata al Gobernador civil y al Ayuntamiento, y

h) nombrar, premiar, corregir, suspender y separar a los Agentes, Guardias y demás miembros del Cuerpo de Policia municipal que usen armas, respecto de todos los cuales corresponde al Alcalde la Jefatura dirccta, sin perjuicio de las superiores atribuciones del Gobernador civil. 
Las Ordenanzas municipales de Barcelona, de 1947, contienen en su Libro II proliferadas normas de Policía urbana que abarcan las solemnidades y festejos, hospedajes y subsistencia, construcciones, circulación, ocupación de la vía pública y diversiones en la misma, industrias callejeras, columnas anunciadoras, instalaciones industriales, policia sanitaria y obligaciones de los ciudadanos en los paseos, jardines, parques y bosques públicos municipales.

En esas disposiciones se condensa la actividad de policia, tanto "previsora" como "sancionadora", constantemente estimulada por el Ayuntamiento para coordinar-como requiere la recia tradición barcelonesa incorporada a la Ciudad modernalos derechos e intereses particulares con el progreso de la comunidad cívica y mantener, mejorar y acrecentar la prestación regular y continua de servicios públicos en el ambiente de orden característico de nuestra gran urbe.

Aprender esas directrices e interpretarlas ponderadamente es lo que habréis de hacer en la fase de formación y ulteriormente en la de capacitación y perfeccionamiento. Pero, por de pronto, considerad con cuánta razón se ha dicho en términos armonitivos que los funcionarios, además de las preocupaciones inherentes a todo hombre que vive de su trabajo y que tiene que atender sus necesidades y las de su familia con unos ingresos, han de llevar consigo una "dotación espiritual".

En la XV Semana Social celebrada en 1955 en la Universidad Pontificia de Salamanca, fué tema de estudio la umoral profesionaln, cuestión de gran importancia según la carta del Secretario sustituto de Estado de Su Santidad, Monseñor Dell' Aqua, en la que analizaba el concepto de profesión como actividad personal realizada en bien de la comunidad con un fin trascendente, y abrazada bajo la dirección de una vocación.

La vocación ha de estar impulsada por el primero de los deberes que señala el artículo 74 del Reglamento de Funcionarios de Administración local, entre los comunes a todos los. funcionarios y con estas palabras: "Cumplir con espiritu de celo y exacta disciplina la misión encomendada, y cooperar al mejoramien- 
to de los servicios y a ia consecución de los fines de la Corporación".

Sea vuestro lema obediencia $y$ disciplina. Obedecer es una alegría, dice León Degrelle, porque es una forma de darse conscientemente. La sociedad humana no es una nube de mosquitos encarnizados y alocados, lanzados al viento según su interés $y$ su humor, sino un complejo sensible, que la anarquía convierte en estéril o peligroso, mientras que el orden y la armonía le dan posibilidades ilimitadas.

Libertad y obediencia no son postulados antitéticos, sino que se complementan recíprocamente. "Libertad obediente", escribe Sassone. Mandar es un deber duro y triste - añade-, y obedecer es un deber que cuando no resulte cómodo y alegre, podrá parecerlo si se ejercita con amor.

San Ignacio recomienda como ejercicio ibuscar razones para defender lo que manda el superior, y no para improbarlo". Obedecer $\sin$ inquirir, dice el Santo, y agrega: aquel monje hizo muy bien en regar durante un año un palo seco, y el Superior hizo bien en probar al súbdito con aquel mandato, aunque nadie afirmara que aquello fué un descubrimiento agronómico.

De nada serviría el poder de mandar - arguye García Oviedo- si no fuese asistido del deber de subordinación o sumisión en que se funda el escalonamiento jerárquico.

La indisciplina quebranta el orden interno de la Administración y se origina por faltas disciplinarias de los funcionarios en el ejercicio de su cargo, que afectan a la propia esencia de la ordenación administrativa.

Entendemos por disciplina, en sentido amplio, el conjunto de reglas y prescripciones relativas al método de vivir, y en cuanto a nuestro examen, el freno legal y reglamentario por el que se dirigen y gobiernar los Cuerpos armados, con respeto profundo a invioiable a sus Ordenanzas, cuyos preceptos requieren la enseñanza, como docencia, y la instrucción como adiestramiento.

Algunos aplican al primer concepto el dictado de disciplina interna y al segundo el de policía externa. En parecida dirección del pensamiento rabria encauzar astas astimaciores luacia 
lo que el Director del Instituto de Estudios de Administración Local, Sr. Ruiz del Castillo, intituló "Técnica y moral de la función pública desde el punto de vista de una filosofía de la cultura", y nosotros hemos acogido bajo el epígrafe de "Carácter moral y disciplinario de la función pública».

La disciplina se establece, se mantiene y se fomenta, partiendo de la organización jerárquica, mediante la celosa vigilancia de los Jefes, la consecuente formación del Cuerpo, el cumplimiento de las Ordenes del día, las amonestaciones y sanciones, los premios y las menciones, según proceda, como advierte mi ilustre colega y caracterizado orientador y enaltecedor de los Cuerpos de la Policía municipal, Gallego y Burín.

Los sistemas de racionalización del trabajo ensayados desde los tiempos de Taylor y Fayol, hasta los del gobierno de empresa que recapitulan y modernizan autores como Jean Chevalier, el arte de mandar forma parte del ejercicio de la función administrativa que tiene por objeto estimular las actividades, coordinar los esfuerzos y mantener la armonía entre los distintos servicios; lo cual implica, en consecuencia, someter la capacidad profesional de los Agentes a la capacidad administrativa de los Jefes.

El leit motiv de la organización continúa siendo, lógicamente, la "relación mando-obediencia» disciplinariamente jerarquizada, lo que entraña, por fortuna, el prevalecimiento del espiritu del hombre sobre la materia de la máquina.

Esas dos vías de conducta, la obediencia y la disciplina, os harán sentir la "autoridad sobre sí mismo», que es la más difícil de todas, y este venturoso arranque vocacional os moverá al cumplimiento de los demás deberes generales: asistir puntualmente al lugar del servicio y permanecer en él durante el horario señalado; observar sigilo escrupuloso respecto de los asuntos que conozcáis por razón del cargo; guardar la lealtad a las Instituciones y respeto y obediencia a las Autoridades y superiores jerárquicos; tratar con esmero y cortesía al público y velar por vuestro propio prestigio.

De vosotros dependerá, en buena parte, que seáis ciertamente un Cuerpo infundido de espíritu, mediante el ejercicio obe- 
diente y disciplinado de viestros deberes, que sor el hilo invisible de la función de policia municipal que habéis de realizar. Para hacerlo asi, recordad las frases alentadoras que, al frente del Ministerio de la Gobernación, del que depende la Policía gubernativa, dirigió a otros funcionarios en $1943 \mathrm{D}$. Blas Pérez Gonzá:ez: "Defendéis un interés de la Ley, el interés de la justicia ; defendéis, en definitiva, algo superior a todos los intereses mezquinos: el bien de la comunidad politica a las que debéis servirn.

\section{Juan Ignacio Berkejo Gironts \\ Secretario general del Excelentisimo \\ Ayratamiento de Barcelona}

